\title{
Expanding the Role of Synthetic Data at the U.S. Census Bureau
}

\section{9th ISI World Statistics Congress \\ August $28^{\text {th }}, 2013$}

By

Ron S. Jarmin

U.S. Census Bureau

Thomas A. Louis

U.S. Census Bureau and John Hopkins University

Javier Miranda

U.S. Census Bureau

Any opinions and conclusions expressed herein are those of the authors and do not necessarily represent the views of the $U$ S. Census Bureau. All results have been reviewed to ensure that no confidential information is disclosed. 


\section{Tension between Confidentiality and Information Content of Statistical Products}

- NSOs use a wealth of information to fulfill their mandate to provide information about the economy and population including

- Survey collections

- Administrative records

- Individuals often are legally required to provide this information

$-\rightarrow$ High response rates

$\rightarrow$ High quality data

$-\rightarrow$ Structured data (representative of the underlying population)

- NSOs are entrusted to use this information only for statistical purposes, with the explicit understanding that the data are confidential and won't be disclosed

- Disclosure protection often codified in statutes

- Necessarily limiting the types of data products that can be publically released 


\section{Increasing the variety and information in statistical}

products while maintaining confidentiality

- Statistical products are constructed via suitable aggregation of confidential survey, census or administrative microdata

- Products cannot reveal data for individual persons, households or firms

- However, products constructed from microdata so that re-identification is sufficiently difficult can potentially reveal details and features that traditional aggregations must mask to maintain confidentiality

- One approach to avoid literal masking is to use multiple imputation to fully or partially synthesize key variables or entire datasets

- The resulting "Synthetic" data set can be used to construct safe data products 


\section{Public-Use Synthetic Microdata}

- Some sophisticated users require access to detailed microdata

- The traditional solution was to provide access through

- Anonymized micro data

- Secure environments such as a Research Data Center

- Anonymized microdata is becoming more risky and so restricted access, even in RDCs

- Synthetic datasets can address both concerns, and Census has produced two experimental versions

- SIPP-Synthetic Beta: household data augmented with administrative data

- Synthetic Longitudinal Business Database (SynLBD): synthetic panel of business establishments 


\section{Synthetic Data}

- Synthetic data is the result of a process of data anonymization

- Synthetic data are generated by modeling real data

- Sampling from posterior predictive distribution

- Sequential regression multivariate imputation

- The resulting data tries to preserve as many of the moments of the real data as well as summary aggregates

- Higher confidentiality protection since respondent information is modeled 


\section{Development of Synthetic data products at the Census Bureau}

- Collaboration between academe and Census research staff

- Cornell and Duke Universities, National Institute for Statistical Sciences

- Center for Economic Studies, Center for Disclosure Avoidance Research and Social, Economic and Household Statistics Division

- Partial funding from the National Science Foundation

- Census staff benefit greatly from working with leading academics

- Collaborations resulted in two class of new data products employing synthetic or partially synthetic data

- Synthetic Public Use Micro Datasets

- Online tools 


\section{Synthetic Data to Support Online Tools and Apps}

- Partially Synthetic data allow users to select custom geographies in "OnTheMap"

- $\quad$ Commuting patterns
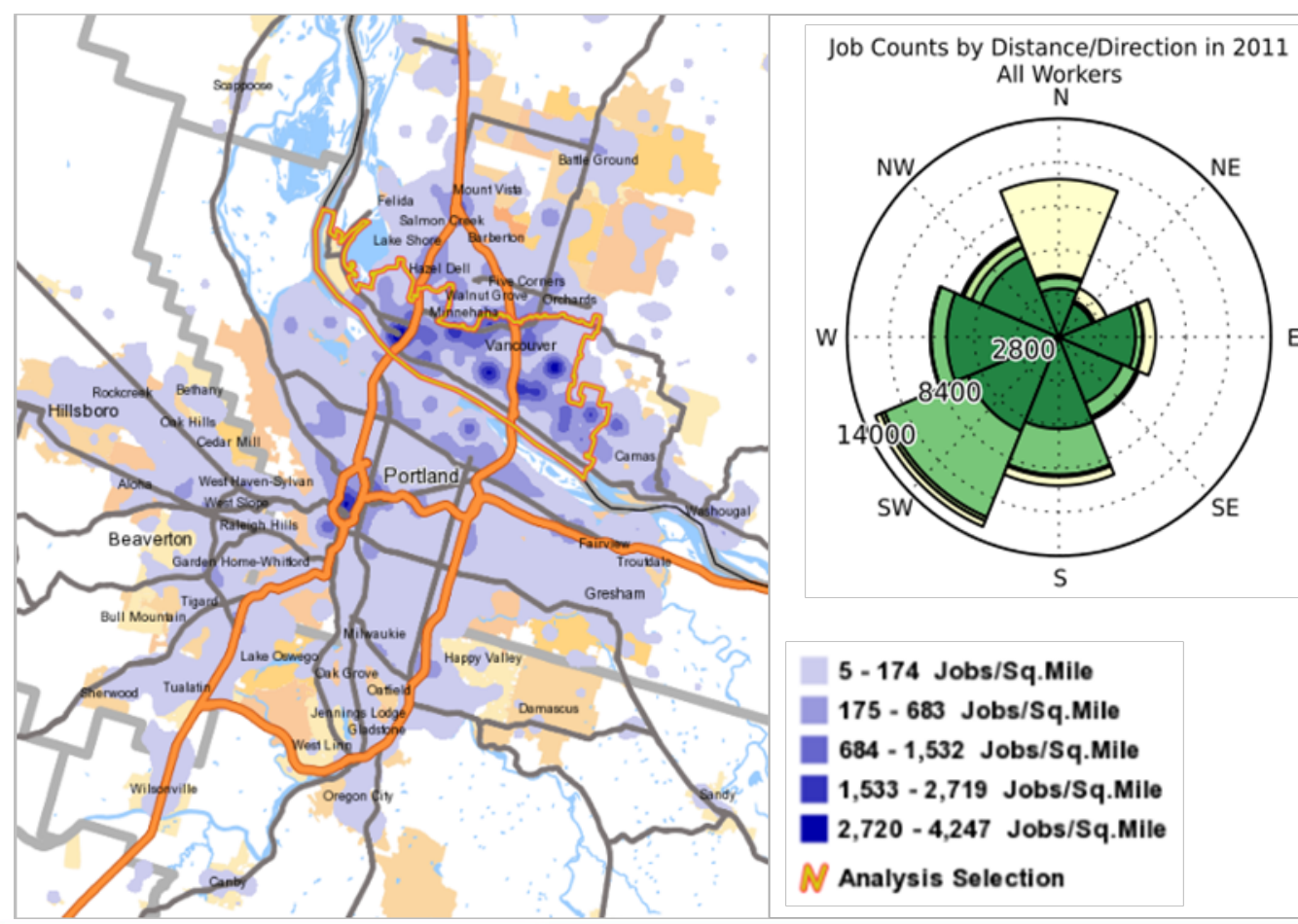

5 - 174 Jobs/Sq.Mile

175 - 683 Jobs/Sq.Mile

$684-1,532$ Jobs/Sq.Mile

1,533 - 2,719 Jobs/Sq.Mile

2,720-4,247 Jobs/Sq.Mile

M Analysis Selection natural disasters

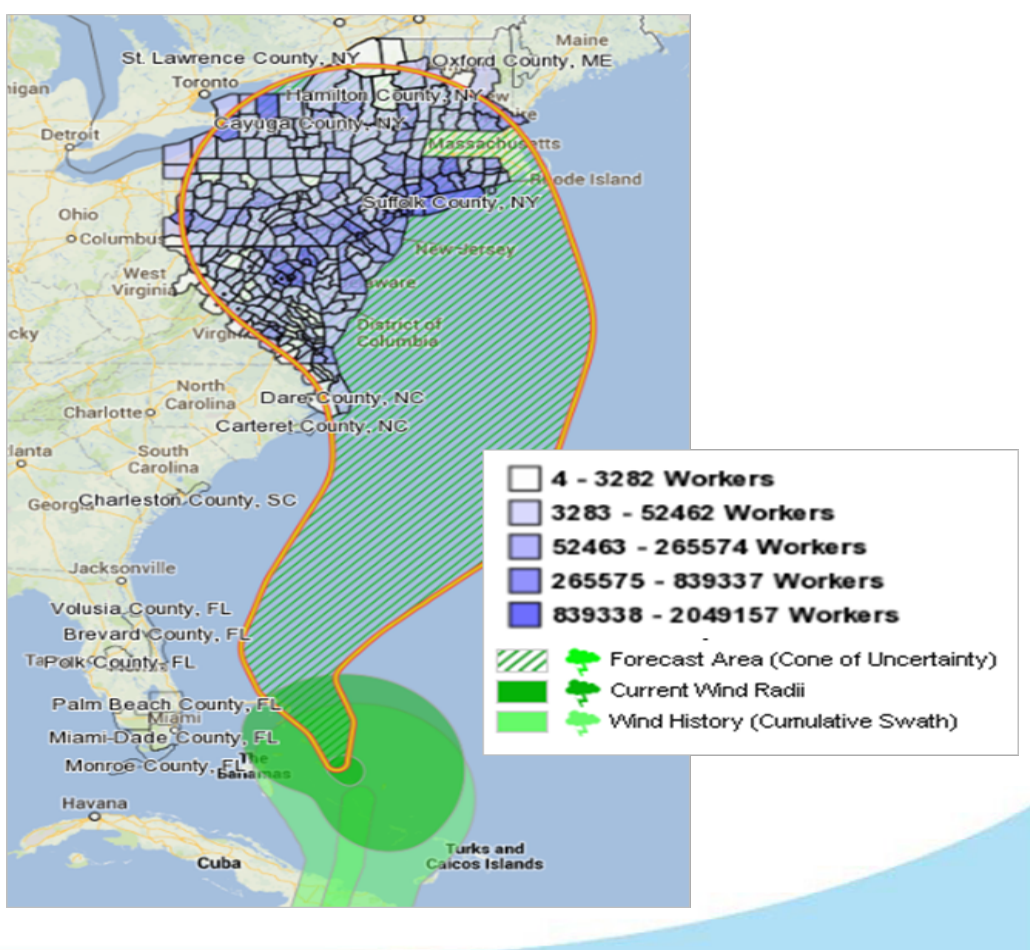




\section{Future of synthetic data in official statistics}

- Data users will access official statistics in apps developed outside of NSOs

- The Census Bureau has an open data API that allows developers to call tabulated estimates from several surveys

- Allowing an API to call from synthetic micro data would allow developers much more latitude in creating apps that give users maximum flexibility

- Synthetic data can play a prominent role in producing publicuse micro datasets as it gets more difficult to release anonymized data that protects confidentiality 


\section{Challenges}

- Synthetic data aren't valid for all analysis goals, and determining validity can be challenging

- Users may not accept or "trust" synthetic data

- On the other hand, users may put too much faith in estimates from synthetic data, taking the values as "truth"

- Already observed for multi-year estimates from the American Community Survey

- Statistical inferences require additional computations, requiring a higher level of user sophistication or flexible algorithms

- NSOs may not have sufficient staff with the training and skills to develop, deploy and support synthetic data products 


\section{Next Steps}

- Research and development of synthetic data products continues

- SynLBD v3 will be expanded to include firm characteristics and full time coverage

- NSOs need to pay special attention to educating the public on correct use of synthetic data and to make sure they know when they are using it

- This might require developing software code to facilitate correct computation of statistical inferences

- Partnerships with academe should be reinforced

- Mutually beneficial and key to developing internal human capital

- Universities can develop courses targeted at NSO staff

- More aggressively explore partnerships with private industry to develop easy to use, multi-platform synthetic data applications

- Resources are limited, but interest and potential are sufficiently high that public/private partnerships are possible 